\title{
Spinal metastasis of parotid acinic cell carcinoma followed by intradural extramedullary recurrence: illustrative case
}

\author{
Po Hsiang (Shawn) Yuan, BSc, ${ }^{1}$ Lukas Grassner, MD, PhD, ${ }^{2-4}$ Charles Fisher, MD, MHSc, FRCSC, ${ }^{5}$ and \\ Nicolas Dea, MD, MSc, FRCSC ${ }^{4}$

\begin{abstract}
${ }^{1}$ Faculty of Medicine, University of British Columbia, Vancouver, British Columbia, Canada; ${ }^{2}$ Department of Neurosurgery, Medical University Innsbruck, Innsbruck, Austria; ${ }^{3}$ Institute of Molecular Regenerative Medicine, Spinal Cord Injury and Tissue Regeneration Center Salzburg, Paracelsus Medical Universit, Salzburg, Austria; ${ }^{4}$ Division of Neurosurgery, Department of Surgery, University of British Columbia, Vancouver, British Columbia, Canada; and ${ }^{5}$ Vancouver Spine Surgery Institute, Department of Orthopaedics, University of British Columbia, Vancouver, British Columbia, Canada
\end{abstract}

BACKGROUND The diagnosis and management of acinic cell carcinoma (ACC) is often challenging given its similarity to benign tumors, high incidences of late recurrence and distant metastasis, and tendency to be resistant to systemic chemotherapy. A primary parotid ACC resulting in an intradural extramedullary mass has not been reported.

OBSERVATIONS The authors describe such a case that presented as a progressive cervical myelopathy 29 years after initial diagnosis. The tumor, located at the $\mathrm{C} 2-\mathrm{C} 3$ level, infiltrated the dura and contained both extradural and intradural components. This occurred 18 months after the incomplete resection of an extradural metastasis at the same location.

LESSONS Although intracranial and extradural metastases of various primary malignancies are well reported, secondary spinal intradural malignancies are rare. As a result, there are no established guidelines for the surgical management of intradural extramedullary metastases and prognosis may be difficult to establish. In this case, treatment options were limited because systemic therapy options had been exhausted and repeated radiation to the area was not recommended. We report on this case to highlight the clinical course of a rare local recurrence after spinal metastasis leading to an intradural extramedullary tumor and to show that surgical intervention can lead to improvement of neurological symptoms.

https://thejns.org/doi/abs/10.3171/CASE21591

KEYWORDS intradural extramedullary; metastasis; parotid tumor; acinic cell carcinoma; myelopathy

Parotid acinic cell carcinoma (ACC) is a rare malignancy of the salivary glands that comprises $6 \%-8 \%$ of all salivary gland neoplasms. ${ }^{1}$ It has a high rate of metastasis and significant tendency for local and late ( $>30$ years) recurrence..$^{1-3}$ The distant spread of salivary gland ACCs occurs in approximately $12 \%$ of patients and is associated with poor survival rates. ${ }^{1}$ The 2 most common metastatic sites of parotid ACCs are lungs and bones, whereas metastases to the cavernous sinus, spine, sternum, orbit, liver, and skin have also been reported. ${ }^{1-3}$

The significant morbidity associated with spinal cord compression secondary to cancer is well documented. ${ }^{4,5}$ However, in almost all cases of spinal cord compression from malignant metastasis, the tumor is located in the epidural space, ${ }^{4,5}$ It is rare to observe an intradural extramedullary (IDEM) metastatic neoplasm and only a handful of such cases have been reported. ${ }^{6-12}$ These cases describe primary malignancies from the breast, kidneys, adrenal glands, esophagus, and lungs. ${ }^{6-13}$ There is not only a lack of guidance toward the surgical management of such cases, but also challenges around diagnosis given low pretest likelihood. We aim to advance our understanding of IDEM malignancies by presenting the first report of a primary parotid ACC resulting in a symptomatic IDEM mass. Although surgical intervention may not be indicated in advanced widespread malignancy, the severely limited treatment options in this case (no further systemic chemotherapy available and no possible repeat radiation to the area), as well as the indolent nature of the primary malignancy, prompted surgical consideration.

ABBREVIATIONS ACC = acinic cell carcinoma; IDEM = intradural extramedullary; MRI = magnetic resonance imaging INCLUDE WHEN CITING Published December 20, 2021; DOI: 10.3171/CASE21591.

SUBMITTED October 15, 2021. ACCEPTED November 10, 2021.

(c) 2021 The authors, CC BY-NC-ND 4.0 (http://creativecommons.org/licenses/by-nc-nd/4.0/). 


\section{Illustrative Case \\ History}

A 79-year-old male with a complicated 29-year history of widespread metastatic parotid ACC presented to the emergency department complaining of a 10-day history of numbness in both hands, worsening dexterity, and deterioration of balance to the point where he was unable to stand independently, symptoms consistent with progressive cervical myelopathy.

The patient was originally diagnosed with left parotid acinar cell carcinoma in 1992 that was treated with total surgical resection, neck dissection, and postoperative radiotherapy and chemotherapy. He remained asymptomatic until local recurrence in 1998 that was treated with further radiation and chemotherapy. Multiple sites of metastases (spine, lung, cerebellum) were identified over the years through regular oncology visits. Because there was no compression and no neurological symptoms, the patient declined surgery and opted for radiation therapy to his cervical and thoracic spine with palliative intent. The systemic disease was managed for decades with radiation and chemotherapy alone.

The patient remained clinically and radiographically stable until 2019 when he presented with a painful lump on his back at the T6 level that on magnetic resonance imaging (MRI) revealed an epidural mass touching the spinal cord. Routine monitoring with the patient's oncologist later revealed disease progression causing a pathologic fracture at the C2 level (Fig. 1). After several months of conservative management, the patient's worsening mechanical neck pain led to surgical intervention via occipitocervical fusion.

A C2-C3 laminectomy with spinal cord decompression and occiputC6 instrument fusion was performed (Fig. 1E) along with debulking of the tumor. Intraoperatively, the tumor was noted to be entirely extradural and extended from $\mathrm{C} 1$ to $\mathrm{C} 3$; a specimen was later confirmed histologically to be metastatic parotid ACC. There were no intraoperative complications, specifically dural tear. The patient was discharged with no neurological deficits, mobilizing well, and without neck pain. No postoperative MRI was done because of clinical improvement and would not have altered management as further radiotherapy was not an option due to having previously received maximal radiation to this area.

Twenty months after his cervical surgery and receiving 2 cycles of chemotherapy during this period, the patient presented to the emergency department with a 10-day history of rapidly worsening cervical myelopathy and was evaluated by the spine surgical service.

\section{Investigations}

An MRI performed showed cord compression caused by an advancing tumor in the upper cervical region (Fig. 2A and B). An intradural component was not initially suspected but the lack of a curtain sign (caused by the displacement of the posterior longitudinal ligament) (Fig. 2B) prompted suspicion that the tumor was not solely extradural. ${ }^{14,15}$ On further review of the gadolinium-enhanced T1-weighted MRI, an intradural component was strongly suspected and preoperative planning was altered accordingly.

\section{Surgical Procedure}

Because the patient had good functional status prior to the onset of rapidly progressive myelopathy, slowly advancing systemic disease, and no further radiation therapy was possible to the area, he was offered a surgical decompression.
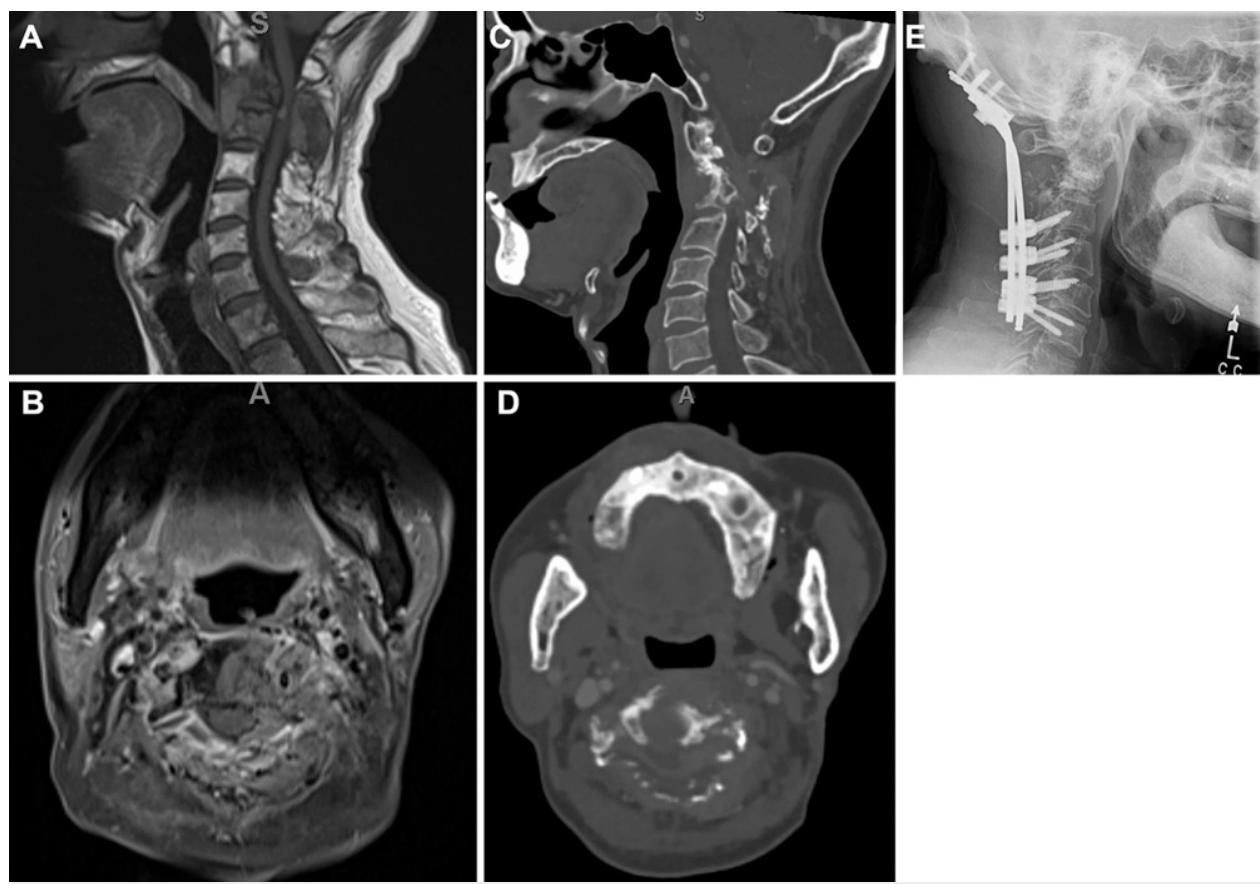

FIG. 1. A: Sagittal T1-weighted MRI with intravenous gadolinium contrast of the cervical spine showing an extradural tumor at the $\mathrm{C} 2-\mathrm{C} 3$ level, prior to any surgical management. B: Axial view of panel A at the C2 level showing compression of the spinal cord. C: Sagittal computed tomography (CT) of the cervical spine showing pathologic fracture of $C 2$, prior to any surgical management. D: Axial view of panel $C$ at the $C 2$ level. $\mathrm{E}$ : Lateral radiograph of the cervical spine post-tumor debulking and instrument fusion from occiput-C6. 

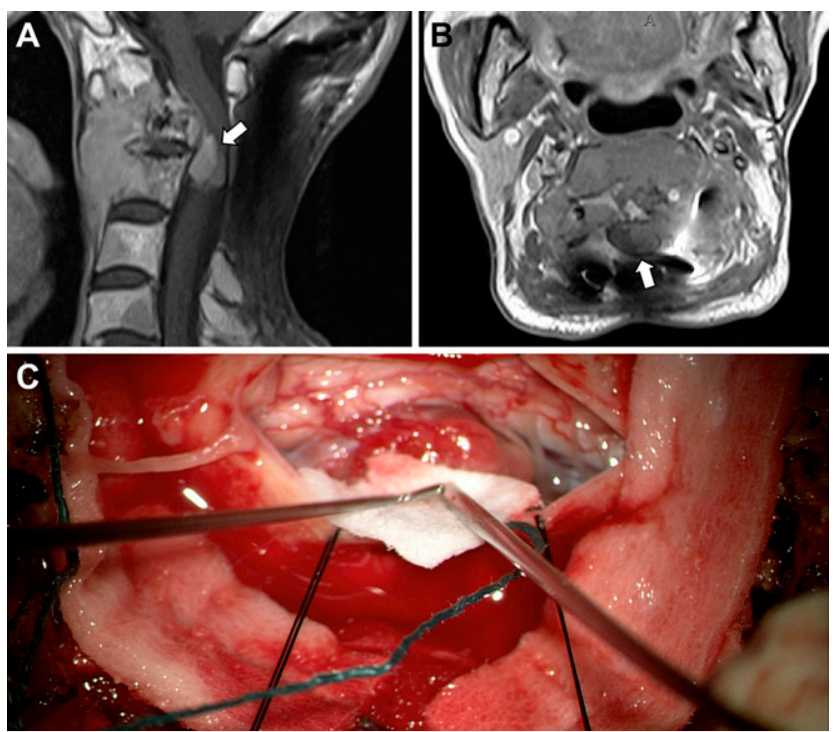

FIG. 2. A: Sagittal T1-weighted MRI with intravenous gadolinium of an intradural extramedullary tumor at the C2-C3 level 18 months after incomplete resection of an extradural metastasis of parotid acinic cell carcinoma. Arrow indicates the interface between extradural and intradural components of the tumor. B: Axial view of panel A at the C3 level showing an intradural extramedullary tumor compressing the spinal cord causing myelopathic symptoms. Arrow indicates the lack of a curtain sign, which would be present in an extradural mass. C: Intraoperative picture of a midline posterior durotomy at the cervical level demonstrating an intradural extramedullary tumor.

The goal of the procedure was to restore neurological function through intradural tumor debulking and dura repair and to replace the failing occiput-C6 fusion hardware. A C1 laminectomy was performed to expose normal dura and an epidural plane was developed through the scar down to the C4 level. An intraoperative ultrasound confirmed the tumor was partly intradural. The extradural portion of the tumor was first debulked posterolaterally. Using the intraoperative microscope, a midline durotomy was then made and the tumor was exposed (Fig. 2). After some debulking, it was apparent that the C3 metastasis had an intradural extension through the C3 nerve root sleeve. Through microsurgical arachnoid dissection, a plane was identified between the cord and the tumor. The C3 dorsal nerve root was infiltrated with tumor so it was cut intradurally. After excision of the tumor, the cord was decompressed, pulsating well, and the tumor entry zone at the nerve root was exposed intradurally. The nerve root exit zone was closed from inside the dura with a running suture and an on lay patch. After irrigation, the dura was closed with a running suture in a watertight fashion. The occipital plate, rods, and set screws were replaced after repositioning the patient for good alignment.

\section{Postoperative Course}

Postoperatively, the patient did well and had $5 / 5$ motor strength in all extremities although some lingering proprioceptive deficiencies affected mobility. The patient reported some decrease in hand numbness and was able to mobilize with a 2-wheel walker. He was then transferred to a rehabilitation facility for safe discharge home at a later date. The intraoperative specimen sent for analysis was confirmed to be primary ACC malignant metastasis as suspected.

\section{Discussion}

IDEM spinal metastases from non-central nervous system primary malignancies are extremely rare and published case reports describe these cases to present as myelopathy, radiculopathy, conus, herniated disc, transverse myelitis, or cauda equina syndrome..$^{6-13,16}$ This often occurs late in the disease course and presentations may be subtle; magnetic resonance imaging with postcontrast gadolinium T1weighted sequencing has been shown to be helpful in detecting IDEM metastases in patients with unexplained neurological symptoms who have an oncological history. ${ }^{14,15,17}$ Palliative surgical management is sometimes warranted in IDEM tumors for symptomatic relief and preservation of neurological function. ${ }^{5,6,11}$ The surgical decision is often impacted by the aggressivity of the primary malignancy and overall prognosis.

\section{Observations}

In this patient, the incomplete resection of an extradural metastatic parotid ACC resulted in a symptomatic local recurrence with intradural extension 18 months later. Initially suspected on T1weighted MRI with gadolinium, this was corroborated by an intraoperative ultrasound and the invasion of the $\mathrm{C} 3$ nerve root sleeve seen intraoperatively. To the best of our knowledge, there is only 1 previously published case of an IDEM metastasis involving an extradural or bony component suggestive of direct invasion. ${ }^{11}$

The patient's age-appropriate baseline functional status guided the decision to offer surgical intervention despite an unclear prognosis and severely limited postoperative treatment options. After excision of the intradural components and debulking of the extradural components, there was recovery of neurological function.

\section{Lessons}

Metastasis to the vertebral column occurs in $3 \%-5 \%$ of all malignancies and only $6 \%$ of these vertebral metastases have an intradural component. ${ }^{11,13}$ The current understanding of IDEM metastasis generally revolves around one or a combination of the 5 following mechanisms: (1) hematogenous seeding of malignant cells via the arterial system; (2) hematogenous seeding via Batson venous plexus; (3) local invasion of the dura; (4) drop metastasis or leptomeningeal spread via the subarachnoid space; and (5) spread from the perineural lymphatics. ${ }^{11}$ It is thought that the most common mechanism is from leptomeningeal spread, because $>50 \%$ of intracranial malignant tumors result in detectable neoplastic cells in the cerebrospinal fluid. ${ }^{11,19}$ Although there was coexisting intracranial disease in the cerebellum of this patient, this was likely not the mechanism responsible for the IDEM tumor observed. We believe that it was rather a direct dural infiltration from a previously treated C3 bony metastasis. The direct invasion through the $\mathrm{C} 3$ nerve root sleeve seen intraoperatively supports this. There is also the possibility of existing but undetected intradural components during the initial cervical surgery. However, during the initial operation, the dura was visualized to be intact and there was clear epidural compression correlating with the preoperative imaging (Fig. 1). As such, we do not believe that there was any intradural tumor at this time.

The surgical decision was guided largely by the patient's baseline function. Previous literature reports that the 6-week postoperative health-related quality of life is not significantly impacted by the 
long-term outcomes of metastatic spine disease and that baseline functional status should take priority in the surgical decision-making process. ${ }^{18}$ The management of IDEM parotid ACC metastasis is further complicated by the lack of consensus regarding the role and efficacy of radiation and chemotherapy. ${ }^{1-3}$ In this case, systemic therapy options had been exhausted and repeat radiation to the area was not recommended.

Many parotid ACCs are considered chemotherapy resistant given its slow metabolism. ${ }^{2}$ The tendency of even low-grade malignancies to metastasize and high incidence of occult disease also contribute to the challenging nature of managing parotid ACCs. ${ }^{1}$ Despite an encouraging 5 -year $91 \%$ and 10 -year $88 \%$ survival rate for primary parotid ACCs, distant spread occurs in $12 \%$ of patients and is associated with poor survival and prognosis., ${ }^{1,2}$ Other poor prognostic factors include high-grade histology, frequent mitoses, regional or distant metastasis upon presentation, age $>30$ years, neural invasion, incomplete excision, male gender, and pleomorphism, many of which apply to this presented case. ${ }^{2}$ Even if the disease is stable and other metastatic lesions are not detected after total ACC IDEM tumor resection, long-term follow-up is crucial given the high likelihood of recurrence and latent or late metastasis, all seen in the clinical course of our patient. ${ }^{1-3}$

In conclusion, there is very limited literature of metastatic malignancies resulting in IDEM neoplasms and no previously published reports of such pathology involving metastatic parotid ACC. This limits our understanding of the diagnosis and management of secondary IDEM malignancies. We aim to advance this knowledge by reporting a case in which the initial incomplete resection of a bony metastasis of a primary parotid ACC at the C3 level resulted in local disease progression and infiltration of the dura 18 months later. Although follow-up is limited, after undergoing microsurgical resection of the intradural tumor, the patient reported restoration of strength, coordination, and balance.

\section{References}

1. Cavaliere M, De Luca P, Scarpa A, et al. Acinic cell carcinoma of the parotid gland: from pathogenesis to management: a literature review. Eur Arch Otorhinolaryngol. 2020;277(10):2673-2679.

2. Al-Zaher N, Obeid A, Al-Salam S, Al-Kayyali BS. Acinic cell carcinoma of the salivary glands: a literature review. Hematol Oncol Stem Cell Ther. 2009;2(1):259-264.

3. Godwin JT, Foote FW Jr, Frazell EL. Acinic cell adenocarcinoma of the parotid gland; report of twenty-seven cases. Am J Pathol. 1954;30(3):465-477.

4. Bach F, Larsen BH, Rohde K, et al. Metastatic spinal cord compression. Occurrence, symptoms, clinical presentations and prognosis in 398 patients with spinal cord compression. Acta Neurochir (Wien). 1990;107(1-2):37-43.

5. Helweg-Larsen S. Clinical outcome in metastatic spinal cord compression. A prospective study of 153 patients. Acta Neurol Scand. 1996;94(4):269-275.

6. Perrin RG, Livingston KE, Aarabi B. Intradural extramedullary spinal metastasis. A report of 10 cases. J Neurosurg. 1982;56(6):835-837.

7. Beehler E. Metastasis to spinal subdural space. Report of case. Bull Los Angel Neuro Soc. 1960;25:44-47.
8. Feiring $\mathrm{EH}$, Hubbard JH Jr. Spinal cord compression resulting from intradural carcinoma. Report of 2 cases. J Neurosurg. 1965;23(6): 635-638.

9. Hirsh LF, Finneson BE. Intradural sacral nerve root metastasis mimicking herniated disc. Case report. J Neurosurg. 1978;49(5): 764-768.

10. Rogers L, Heard G. Intrathecal spinal metastases (rare tumours). Br J Surg. 1958;45(192):317-320.

11. Land CF, Bowden BD, Morpeth BG, DeVine JG. Intradural extramedullary metastasis: a review of literature and case report. Spinal Cord Ser Cases. 2019;5(1):41.

12. Lee $\mathrm{CH}$, Kim KJ, Hyun SJ, Jahng TA, Kim HJ. Intradural extramedullary metastasis of small cell lung cancer: a case report. Korean J Spine. 2012;9(3):293-296.

13. Lin TK, Chen SM, Jung SM. Solitary intradural extramedullary metastasis of renal cell carcinoma to the conus medullaris. Kaohsiung J Med Sci. 2011;27(1):45-48.

14. Shah LM, Salzman KL. Imaging of spinal metastatic disease. Int $J$ Surg Oncol. 2011;2011:769753.

15. Sze G, Abramson A, Krol G, et al. Gadolinium-DTPA in the evaluation of intradural extramedullary spinal disease. AJR Am J Roentgenol. 1988;150(4):911-921.

16. Ding D, Fullard M, Jarrell HS, Jones DE. Intramedullary spinal cord metastasis from salivary ductal carcinoma of the parotid gland mimicking transverse myelitis in a patient with radiologically isolated syndrome. J Neurol Sci. 2014;336(1-2):265-268.

17. Frey I, Le Breton C, Lefkopoulos A, et al. Intradural extramedullary spinal canal secondary neoplasms: MR findings in 30 patients. Eur Radiol. 1998;8(7):1187-1192.

18. Dea N, Versteeg AL, Sahgal A, et al. Metastatic spine disease: should patients with short life expectancy be denied surgical care? An international retrospective cohort study. Neurosurgery. 2020;87(2):303-311.

19. Knafo S, Pallud J, Le Rhun E, et al. Intradural extramedullary spinal metastases of non-neurogenic origin: a distinct clinical entity or a subtype of leptomeningeal metastasis? A case-control study. Neurosurgery. 2013;73(6):923-931, discussion 932.

\section{Disclosures}

Dr. Fisher reported personal fees from Medtronic, personal fees from Nuvasive, grants from OREF, other from Medtronic, and other from AO Spine outside the submitted work. No other disclosures were reported.

\section{Author Contributions}

Conception and design: all authors. Acquisition of data: Dea, Yuan, Grassner. Analysis and interpretation of data: all authors. Drafting the article: Yuan, Grassner. Critically revising the article: Dea, Yuan, Grassner. Reviewed submitted version of manuscript: all authors. Approved the final version of the manuscript on behalf of all authors: Dea. Administrative/technical/material support: Yuan. Study supervision: Dea, Grassner, Fisher.

\section{Correspondence}

Nicolas Dea: University of British Columbia, Vancouver, BC, Canada. nicolas.dea@vch.ca. 